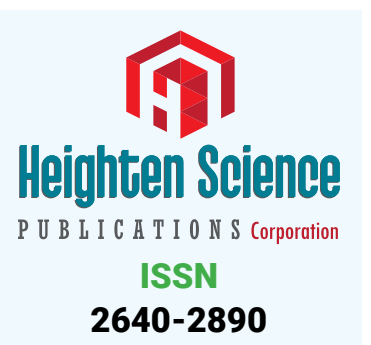

*Address for Correspondence: Andrea Plaikner, MD, Department of Advanced Operative and Oncologic Gynecology, Asklepios Hospital, PaulEhrlich-Straße, 22763 Hamburg, Germany, Tel: 0049401818811641 ;

Email: a.plaikner@asklepios.com

Submitted: 26 April 2019

Approved: 14 May 2019

Published: 15 May 2019

Copyright: @ 2019 Plaikner A, et al. This is an open access article distributed under the Creative Commons Attribution License, which permits unrestricted use, distribution, and reproduction in any medium, provided the original work is properly cited

Keywords: Anti-NMDA receptor encephalitis; Ovarian teratoma

Check for updates
Case Report

\section{Autoimmune encephalitis associated with an ovarian teratoma in a 29-year old woman}

\author{
A Plaikner ${ }^{1}$, A Jacob ${ }^{1}$, J Rother ${ }^{2}$ and C Kohler ${ }^{3}$ \\ 'Department of Advanced Operative and Oncologic Gynecology, Asklepios Hospital, Paul-Ehrlich- \\ Straße 1, 22763 Hamburg, Germany \\ 2Department of Neurology, Asklepios Hospital, Paul-Ehrlich-Straße 1, 22763 Hamburg, Germany \\ ${ }^{3}$ Department of Advanced Operative and Oncologic Gynecology, Asklepios Hospital, Paul-Ehrlich- \\ Straße 1, 22763 Hamburg, \& Department of Gynecology, University of Cologne, Kerpener Straße 34, \\ 50931, Medical Faculty, Cologne, Germany
}

\section{Abstract}

NMDA receptor encephalitis is a rare disease first described in 2007. Anti-NMDA receptor encephalitis affects mostly young women as neoplasms, mostly ovarian teratomas, are the underlying cause. The disease is caused by antibodies binding to extracellular epitopes of neuronal cell-surface, which leads to an internalization of NMDA-receptors. The characteristic syndrome of patients with anti-NMDAR as well as its recovery follows a certain pattern. Treatment includes immunotherapy and removal of the immunologic trigger. This case report describes a young woman with anti-NMDA receptor encephalitis caused by an ovarian teratoma.

\section{Introduction}

Encephalitis associated with antibodies against N-methyl-D-aspartate receptor (NMDAR) was first described in 2007 [1,2]. While annual incidence of all types of encephalitis is approximately 5 to 8 per 100.000 , the exact incidence of this specific autoimmune encephalitis is unknown [2,3]. Autoimmune reactions are the third most common cause of encephalitis after infections (mostly viral) and acute disseminated encephalomyelitis (usually postinfectious) [2].

The most frequent form of autoimmune encephalitis is caused by antibodies against NMDAR [4]. Anti-NMDAR encephalitis affects mostly women (4:1), the median age is 21. Up to $58 \%$ of affected young women have an ovarian teratoma, in men and children the association with tumours is less frequent [5]. In a retrospective study anti-NMDAR encephalitis was the reason for $1 \%$ of all admissions in young adults to intensive care units [6]. The distinction between paraneoplastic symptoms (PNS) and autoimmune encephalitis is crucial. Despite similar symptoms pathogenic mechanisms and outcomes are different. PNS are associated with antibodies against intracellular neuronal antigens leading to a cytotoxic T-cell reaction, whereas autoimmune encephalitis is caused by antibodies binding to extracellular epitopes of the neuronal cell-surface. This may also explain better outcomes of patients with autoimmune encephalitis [5].

The characteristic syndrome of patients with anti-NMDAR as well as its recovery follows a certain pattern. $70 \%$ of patients have prodromal symptoms such as headache, fever, nausea, vomiting, diarrhoea or upper respiratory tract symptoms. Within less than two weeks the patients develop psychiatric symptoms like anxiety, insomnia, 
fear, grandiose delusions, hyper-religiosity, mania and paranoia. Short term memory loss is common, but difficult to assess. Further symptoms are a rapid disintegration of language, from reduction of verbal output to frank mutism. In children, the behavioural change can be difficult to detect, temper change, hyperactivity and irritability are common. After this initial phase, decreased responsiveness can alter with agitation. Since psychiatric symptoms are dominant, many patients are admitted to psychiatry under the assumption of acute psychosis which often delays diagnosis. Autonomic manifestations such as hyperthermia, tachycardia or urinary incontinence can occur. Seizures are not uncommon but can be masked by oro-lingual-facial dyskinesia or choreoatetosis [1]. Basic diagnostic assessment includes brain MRI, electroencephalograms (EEG), spinal punction and analysis of cerebrospinal fluid (CSF).

MRI shows normal results in $50 \%$ of cases, the other patients show a mild signal hyperintensity in the hippocampi, cerebellum or cerebral cortex, frontobasal and insular regions, basal ganglia, brainstem and, rarely, the spinal cord. The EEG is abnormal in most cases, showing non-specific, slow and disorganised activity sometimes with electrographic seizures. The cerebrospinal fluid is abnormal in at least $80 \%$ of cases and shows lymphocytic pleocytosis, normal or mildly increased protein concentration and, in $60 \%$ of patients oligoclonal bands. Most patients have intrathecal synthesis of NMDAR antibodies [7]. Brain biopsy is not suitable for the diagnosis of anti-NMDAR encephalitis [1].

The current standard treatment includes immunotherapy and removal of the immunologic trigger, such as teratoma or other tumors, when applicable. A good outcome greatly depends on early removal of the tumor [5]. In addition, most patients are treated with glucocorticoids, intravenous immune globuline or plasma exchange, and - if a clinical response is missing - rituximab and cyclophosphamide [5].

Within four weeks, $53 \%$ of patients show a clinical improvement, $75-81 \%$ reach substantial recovery (mild or no residual symptoms) at 24 months [5]. Up to $25 \%$ remain severely disabled or die from the disease [1]. Management of anti-NMDAR encephalitis should initially focus on immunotherapy and detection and removal of a teratoma or tumour. In young women with suspicion of autoimmune encephalitis an immediate gynaecologic consultation should be initiated. After hospital admission of the patient presented below a systematic review of the literature on this rare disease was done.

\section{Case Presentation}

A 29-year-old patient was admitted after three days of progressive aphasia (bulbar speech, sentence structure disorder) since 3 days. She reported mild prodromal symptoms such as left-sided headache for more than four weeks. Neurological examination was normal besides fluctuating speech disturbance. While word and sentence recognition was not affected, the patient had difficulties forming sentences, had phases of stuttering and mute episodes, where she was not able to answer at all and seemed desperate about her condition.

Cranial MRI and CT imaging showed physiological findings, no stroke or intracranial bleeding was detected. EEG showed normal alpha activity without seizures. Additional examinations, such as electrocardiogram or cranial color-coded duplex sonography demonstrated no pathological findings.

There was no sign of infectious disease. Solely CSF showed the tipical lymphocytic pleocytosis with $27 / \mu \mathrm{l}$ cells, oligoclonal bands and normal protein level. Antibodies against NMDAR were highly positive in the cerebrospinal fluid and blood serum, which in conclusion lead to the diagnosis. Immunotherapy with corticosteroids and intravenous immunoglobulins was started immediately and the patient underwent gynaecological examination. The transvaginal ultrasound showed a tumour of $6 \times 4 \mathrm{~cm}$ of the 
left ovary with mixed echogenity suspicious for teratoma. The patient gave consent to laparoscopic salpingoophorectomy, and the procedure was performed the following day. Final histopathologic examination showed mature teratoma without signs of malignancy. In the following days the patients' condition was fast improving under ongoing therapy with intravenous immunoglobulins ( 30 gr per day on 5 consecutive days). The patient was discharged three days after the operation. At follow-up investigation after 3 months she had completely recovered.

\section{Discussion}

Anti-NMDA receptor encephalitis is a neurologically well-defined autoimmune disease with a progressive clinical course of neurological and psychiatric symptoms. The outcome is favourable, if diagnosis can be made quickly and treatment starts early. Triggers of encephalitis are viral infections (herpes simplex typ 1, west nil virus, enteroviruses and varicella zoster virus), tumors and other unknown factors [2]. The pathogenic role of anti-NMDAR antibodies has been proven in vitro and in vivo [2]. The target is the NR1 subunit of the NMDA receptor [8]. Binding of the antibodies and the receptor leads to internalization of the receptors and their concentration in the postsynaptic membrane decreases [2].

As shown in autopsy and pathological studies, ovarian teratomas can contain tissue of mature and immature neurons expressing NMDA receptors. Inflammatory cell infiltrates are found in tissue samples of patients with anti-NMDAR encephalitis. Therefore teratomas may play a role in triggering the synthesis of such antibodies [2].

Early treatment is crucial for achieving a good outcome. Most patients are treated with glucocorticoids, intravenous immunglobuline or plasma exchange, and if a clinical response is missing, rituximab and/or cyclophosphamide is given [5]. Prompt immunotherapy and complete tumor resection are prerequisites for a favorable outcome $[2,9]$.

The presence of a tumor as underlying cause depends on age, sex, and ethnic background. In $46 \%$ of women a benign or malignant neoplasm can be detected. It is rarely found in girls younger than 12 years (6\%) and male patients (6\%) [2]. Ovarian teratoma is the most common underlying tumor, although extraovarian teratoma (2\%) and other tumors ( $4 \%$, e.g. lung, breast, testis, ovary, uterus, thymus and pancreas) can be found [2]. While neurologists see many cases of anti-NMDAR-encephalitis, the disease is relatively unknown amongst gynaecologists, even though an essential part of the treatment is the removal of tumor.

Anti-NMDAR encephalitis mostly affects women in reproductive age, thus different ways of tumor removal should be discussed to preserve ovarian tissue for fertility. Very often a salpingo-oophorectomy is performed, especially in small lesions where the location of the tumor can't be identified macroscopically. As bilateral teratomas are described in $15 \%$ of cases [10] (Figure 1), it is important to find fertility sparing options for the young patients. Asai et al., showed similar outcomes with cystectomy as with adnexectomy [11]. Jones et al., described a case of a 29-year old women with anti-NMDAR encephalitis and an $8 \mathrm{~mm}$ ovarian lesion, which was selectively removed via ultrasound-guided laparoscopy [9]. Another clinical scenario suggests that patients with no response to first- and second line immunotherapy may benefit from the removal of normal-appearing ovaries. Two identical twin sisters with anti-NMDAR encephalitis did not improve after immunotherapy. One twin received immunotherapy only and died from the disease, her sister accepted bilateral salpingo-oophorectomy and recovered gradually [2]. Theoretically, selective ovarian vein sampling could be undertaken to measure anti-NMDAR antibody concentration, extrapolated from a similar technique that has been successfully used in the diagnosis of androgen producing ovarian tumors not visible with conventional imaging modalities. Alternatively, bilateral ovarian wedge biopsies could be performed, to help identify occult teratomatous tissue [9]. 


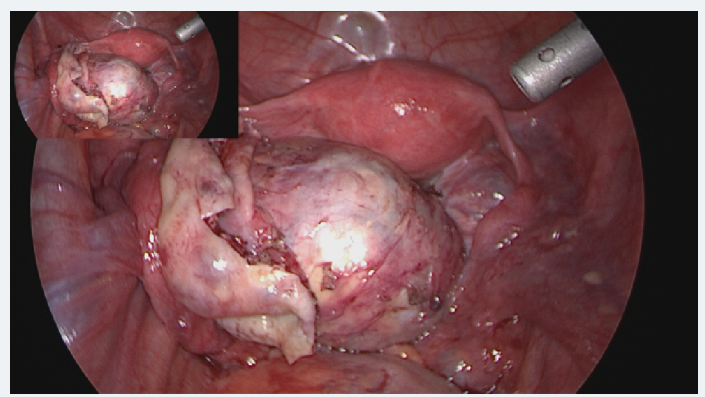

Figure 1: Ovarian tumor.

Adnexectomy should be reserved for cases of suspected immature teratomas and treatment-resistant cases with no sign of teratoma.

Our patient showed typical symptoms for anti-NMDAR encephalitis, she was diagnosed quickly and treated by immunotherapy with corticosteroids and intravenous immunoglobulins as well as laparoscopic salpingo-oophorectomy although the above mentioned studies show that probably an ovary sparing operation would have been sufficient. Our case supports the assumption that a prompt diagnosis and treatment can result in a favorable outcome for the patient. In conclusion a wideer knowledge of this disease amongst gynecologists could be beneficial for patiernt as it might lead to earlier diagnosis and faster treatment.

\section{References}

1. Dalmau J, Lancaster E, Martinez-Hernandez E, Rosenfeld MR, Balice-Gordon R. Clinical experience and laboratory investigations in patients with anti-NMDAR encephalitis. Lancet Neurol. 2011; 10: 6374. Ref.: https://tinyurl.com/y66m2kmr

2. Liu CY, Zhu J, Zheng XY, Ma C, Wang X. Anti-N-Methyl-D-aspartate Receptor Encephalitis: A Severe, Potentially Reversible Autoimmune Encephalitis. Mediators Inflamm. 2017; 6361479. Ref.: https://tinyurl.com/y69ak8pr

3. Granerod J, Ambrose HE, Davies NW, Clewley JP, Walsh AL, et al. Causes of encephalitis and differences in their clinical presentations in England: a multicentre, population-based prospective study. Lancet Infect Dis Dec. 2010; 10: 835-844. Ref.: https://tinyurl.com/y39y4tm5

4. Gable MS, Sheriff H, Dalmau J, Tilley DH, Glaser CA. The frequency of autoimmune N-methyl-Daspartate receptor encephalitis surpasses that of individual viral etiologies in young individuals enrolled in the California Encephalitis Project. Clin Infect Dis Apr. 2012; 54: 899-904. Ref.: https://tinyurl.com/y38emwag

5. Dalmau J, Graus F. Antibody-Mediated Encephalitis. N Engl J Med. 2018; 378: 840-851. Ref.: https://tinyurl.com/yygubeje

6. Pruss H, Dalmau J, Harms L, Holtje M, Ahnert-Hilger G, et al. Retrospective analysis of NMDA receptor antibodies in encephalitis of unknown origin. Neurology. 2010; 75: 1735-1739. Ref.: https://tinyurl.com/y3267stu

7. Pruss $\mathrm{H}$, Dalmau J, Arolt V, Wandinger KP. [Anti-NMDA-receptor encephalitis. An interdisciplinary clinical picture]. Nervenarzt. 2010; 81: 396. 398, 400, passim. Ref.: https://tinyurl.com/y5wm6foy

8. Dalmau J, Gleichman AJ, Hughes EG, Rossi JE, Peng X, et al. Anti-NMDA-receptor encephalitis: case series and analysis of the effects of antibodies. Lancet Neurol. 2008; 7: 1091-1098. Ref.: https://tinyurl.com/yxuep6p5

9. Jones BP, Rees R, Saso S, Stalder C, Smith JR, et al. Ultrasound-guided laparoscopic ovarian preserving surgery to treat anti-NMDA receptor encephalitis. BJOG. 2017; 124: 337-341. Ref.: https://tinyurl.com/y6tzoxp3

10. Naoura I, Didelot A, Walker F, Luton D, Koskas M. Anti-N-methyl-D-aspartate receptor encephalitis complicating ovarian teratomas: a case report. Am J Obstet Gynecol. 2011; 205: e6-8. Ref.: https://tinyurl.com/y6nz7h8q

11. Asai $\mathrm{S}$, Ishimoto $\mathrm{H}$, Yabuno $A$, Asada $\mathrm{H}$, Seki $\mathrm{M}$, et al. Laparoscopic cystectomy of ovarian teratoma in anti-NMDAR encephalitis: 2 case reports. J Minim Invasive Gynecol. 2011; 18:135-137. Ref.: https://tinyurl.com/y3ohsp9v 